\title{
A Self-Consistent Mean Flow Description of the Nonlinear Saturation of the Vortex Shedding in the Cylinder Wake
}

\author{
Vladislav Mantič-Lugo, Cristóbal Arratia, and François Gallaire \\ Laboratory of Fluid Mechanics and Instabilities, École Polytechnique \\ Fédérale de Lausanne. EPFL-STI-IGM-LFMI, CH-1015, Switzerland
}

(Dated: July 29, 2014)

\begin{abstract}
The Bénard-von Karman vortex shedding instability in the wake of a cylinder is perhaps the best known example of a supercritical Hopf bifurcation in fluid dynamics. However, a simplified physical description that accurately accounts for the saturation amplitude of the instability is still missing. Here we present a simple self-consistent model that provides a clear description of the saturation mechanism and quantitatively predicts the saturated amplitude and flow fields. The model is formally constructed by a set of coupled equations governing the mean flow together with its most unstable eigenmode with finite size. The saturation amplitude is determined by requiring the mean flow to be neutrally stable. Without requiring any input from numerical or experimental data, the resolution of the model provides a good prediction of the amplitude and frequency of the vortex shedding, as well as the spatial structure of the mean flow and the Reynolds stress.
\end{abstract}

Simple models are essential to our understanding of complex nonlinear phenomena. The van der Pol oscillator, for example, demonstrates how nonlinear oscillations can be described by the appearance of a limit cycle 1]. In large dimensional systems, however, these simple models do not entirely reveal the mechanisms that determine relevant parameters like the dominant frequency or saturation amplitude. For supercritical instabilities in fluid dynamics, the mean flow has been proposed as a key element to explain the origin of the dominant frequency [2 [5] and the physical mechanism of the saturation process $[5$ [7]. The physical picture thus invoked to understand the saturation is the following: perturbations feeding on an unstable flow induce mean flow modifications that increase while perturbations grow, up to the point where the mean flow becomes neutrally stable and perturbations stop growing and saturate. The present Letter aims at assessing this scenario.

An early formulation of this concept of marginal stability of the mean flow was given by Malkus [8] in the context of turbulent flows. Shortly after, aiming for an equation describing the saturation of supercritical instabilities, Stuart [6] devised a simplified closed system wherein the mean flow was only affected by the Reynolds stress divergence of its leading eigenmode. By further assuming that the eigenmode was given by the unperturbed base flow, Stuart managed to obtain an equation for the saturation amplitude through the exact balance between the dissipation of the perturbation and the energy transfer from the mean flow. It wasn't until after two more years, through a more rigorous perturbative analysis close to threshold, that he mathematically derived an amplitude equation, the Stuart-Landau equation, directly from the Navier-Stokes equations [9].

Despite the beauty and consistency of the multiplescale expansion method, its perturbative nature implies that the spatial structure of the growing unstable mode is in large part fixed by the unperturbed base flow. How- ever, there are cases in which the spatial structure of the saturated mode differs considerably from that of the linear mode, limiting the validity of the usual StuartLandau amplitude equation [10, 11]. This opens the question of whether one can formulate a more accurate prediction of the saturation amplitude by retaining some of the spatial degrees of freedom.

The purpose of the present Letter is to propose a model that physically describes the saturation mechanism of an unstable flow, shedding some light on the nonlinear effects that are relevant for the coupling of the perturbation and the mean flow equations. The coupled equations are solved in a self-consistent way, through a quasi-linear single harmonic approximation of the perturbation, allowing to determine a priori the mean flow and the frequency and structure of the dominant harmonic perturbation, without resorting a posteriori to linear stability analysis of mean flows averaged from DNS or experimental data. In addition, the method yields a prediction of the perturbation amplitude.

A widely studied archetype of a supercritical instability in fluid flows is the flow past a circular cylinder 12], characterized by the non-dimensional Reynolds number $R e=U_{\infty} D / \nu$, where $\nu$ is the kinematic viscosity, $U_{\infty}$ the free-stream velocity and $D$ the cylinder diameter. As the Reynolds number increases, a Hopf bifurcation occurs at $R e \sim 47$ [13] and the flow dynamics changes from a steady symmetric state to an oscillating time periodic configuration presenting the Bénard-von Karman vortex street [14]. The contribution of the fundamental harmonic is dominant compared to the higher harmonics even in the fully saturated regime [10].

Linear stability analyses describes well this instability by predicting the value of the threshold as well as the shedding frequency at threshold [11, 15, 16]. However, as noticed in Refs. [4] and [5], the frequency prediction based on the leading eigenvalue of the base flow does not match the experiments as one departs from threshold. 
In contrast, the stability analysis around the mean flow shows a remarkably good frequency prediction with almost zero growth rate, supporting the validity of Malkus' marginal stability criterion. However, these linear stability analyses cannot provide any information about the perturbation amplitude.

Qualitative aspects of the saturation of the instability are well described by a Stuart-Landau amplitude equation, the coefficients of which could be obtained both empirically [10] and using the multiple-scale expansion, as done by Sipp and Lebedev [17]. However, due to the perturbative nature of its derivation, this model is quantitatively valid only very close to threshold. We therefore focus on the supercritical regime of the flow past a cylinder, for $R e>47$ and propose a non-perturbative quasilinear model coupling the mean flow equation to a linear harmonic disturbance, consistently accounting for the mean flow distortion. In this approach, the mean flow comes as a result of the model instead of being required as an input.

The starting point of the model is the Reynolds decomposition $\boldsymbol{u}(\boldsymbol{x}, t)=\boldsymbol{U}(\boldsymbol{x})+\boldsymbol{u}^{\prime}(\boldsymbol{x}, t)$ of the instantaneous flow in mean $\boldsymbol{U}=\langle\boldsymbol{u}\rangle$ and perturbation $\boldsymbol{u}^{\prime}$, where \langle\rangle denotes time-averaging and where $\left\langle\boldsymbol{u}^{\prime}\right\rangle=0$ by construction. Because of incompressibility, it is understood in what follows that all velocity fields must be divergence free; we will not write this condition explicitly. This decomposition can be introduced in the 2D incompressible Navier-Stokes equations to yield

$$
\begin{aligned}
N(\boldsymbol{U}) & =-\left\langle\left(\boldsymbol{u}^{\prime} \cdot \nabla\right) \boldsymbol{u}^{\prime}\right\rangle \\
\partial_{t} \boldsymbol{u}^{\prime}+L_{\boldsymbol{U}}\left(\boldsymbol{u}^{\prime}\right) & =-\left(\boldsymbol{u}^{\prime} \cdot \nabla\right) \boldsymbol{u}^{\prime}+\left\langle\left(\boldsymbol{u}^{\prime} \cdot \nabla\right) \boldsymbol{u}^{\prime}\right\rangle
\end{aligned}
$$

where

$$
N(\boldsymbol{U}) \equiv(\boldsymbol{U} \cdot \nabla) \boldsymbol{U}+\nabla P-R e^{-1} \Delta \boldsymbol{U},
$$

corresponds to the advective, pressure gradient and diffusive terms of the Navier-Stokes equations (NSE) and $L_{U}\left(\boldsymbol{u}^{\prime}\right)$ is the corresponding operator for the NSE linearized around $\boldsymbol{U}$, i.e.

$$
L_{\boldsymbol{U}}\left(\boldsymbol{u}^{\prime}\right) \equiv(\boldsymbol{U} \cdot \nabla) \boldsymbol{u}^{\prime}+\left(\boldsymbol{u}^{\prime} \cdot \nabla\right) \boldsymbol{U}+\nabla p^{\prime}-R e^{-1} \Delta \boldsymbol{u}^{\prime} .
$$

$P$ and $p^{\prime}$ are the pressure fields required to impose incompressibility on $\boldsymbol{U}$ and $\boldsymbol{u}^{\prime}$, respectively. No approximation has been performed so far.

Different nonlinear effects can be identified in (11). The right hand side (RHS) of (1a) corresponds to minus the Reynolds stress divergence $\left\langle\left(\boldsymbol{u}^{\prime} \cdot \nabla\right) \boldsymbol{u}^{\prime}\right\rangle$, a forcing of $\boldsymbol{u}^{\prime}$ on $\boldsymbol{U}$ which can be also viewed as the body force required for the mean flow $\boldsymbol{U}$ to become a stationary solution of the NSE [5]. The effect of the mean flow $\boldsymbol{U}$ back on the fluctuating part $\boldsymbol{u}^{\prime}$ is contained in the nonlinear advection terms of the linearized operator $L_{\boldsymbol{U}}\left(\boldsymbol{u}^{\prime}\right)$; with $\boldsymbol{U}$ prescribed by experimental or computational data, these are the terms that are taken into account in mean

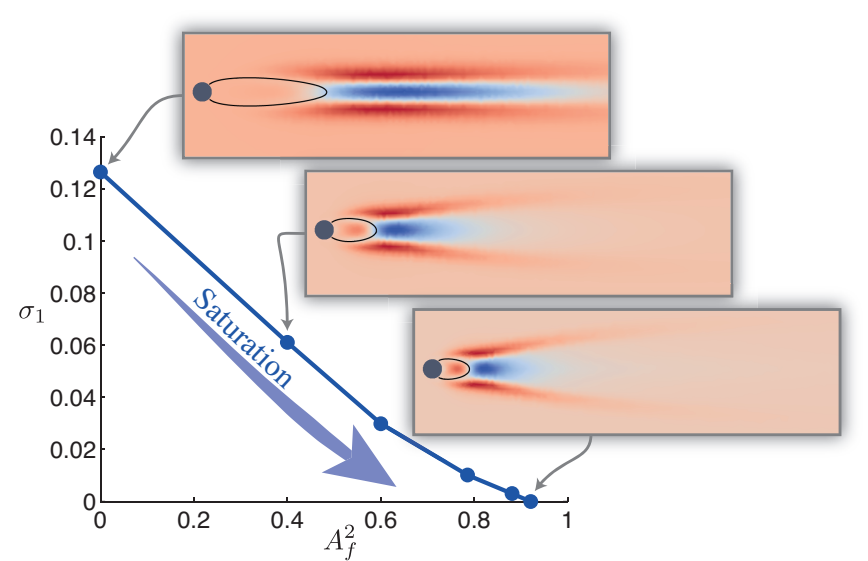

FIG. 1. Growth rate $\sigma_{1}$ for the converged coupled system of equations of the self-consistent model (4) for different Reynolds stress forcing amplitudes $A_{f}^{2}$ at $\mathrm{Re}=100$. The insets show the spatial distribution of the of the divergence of the Reynolds stress in the $x$-direction and the boundary of the recirculation region for different $A_{f}^{2}$, as indicated in the figure.

flow stability analyses. The RHS of (1b) contains the nonlinear terms that allow interactions of $\boldsymbol{u}^{\prime}$ at different frequencies and generate different harmonics. In the present case of the cylinder, the power spectra of the vortex shedding signal is strongly dominated by a single frequency, the fundamental harmonic of the vortex shedding [10]. Neglecting the nonlinear terms in the RHS, Eq. (1b) becomes linear in $\boldsymbol{u}^{\prime}$. The linearized Navier-Stokes operator can be diagonalized and the perturbation can be therefore expanded into the basis of its eigenmodes $\boldsymbol{u}_{n}^{\prime}=\boldsymbol{u}_{n} \exp \left(\lambda_{n} t\right)+\overline{\boldsymbol{u}}_{n} \exp \left(\bar{\lambda}_{n} t\right)$, where the overbar represents the complex conjugate. Focusing on the least stable eigenmode pair $n=1$, one obtains

$$
\begin{aligned}
N(\boldsymbol{U}) & =-2 A^{2} \Re\left(\left(\overline{\boldsymbol{u}}_{1} \cdot \nabla\right) \boldsymbol{u}_{1}\right), \\
\lambda_{1} \boldsymbol{u}_{1}+L_{\boldsymbol{U}}\left(\boldsymbol{u}_{1}\right) & =0
\end{aligned}
$$

where $\boldsymbol{u}_{1}$ is the least stable eigenmode of the model mean flow $\boldsymbol{U}$ as computed from (4b), $\lambda_{1}=\sigma_{1}+i \omega_{1}$ is its associated eigenvalue and $A$ is a real constant that represents the amplitude of the first eigenmode as normalized by its $L^{2}$ norm. $\Re(\cdot)$ in (4a) denotes the real part. In the computation of the Reynolds stress divergence, the time variation associated to the real part of the eigenvalue $\sigma_{1}$ is neglected. Therefore, Eq. (4a) can be seen as a quasistatic approximation of the mean flow in which the slow time scale dynamics associated to the growth of the unstable mode is slaved to the amplitude $A$, which may be then treated as an external parameter.

The straightforward solution of (4) for $A=0$, corresponds to the base flow $\boldsymbol{U}_{B}$, i.e. the stationary solution of the NSE together with its corresponding unstable eigenmode $\boldsymbol{u}_{1, B}$, which represents the initial perturbation growing at a rate $\sigma_{1, B}$ and frequency $\omega_{1, B}$. If the 

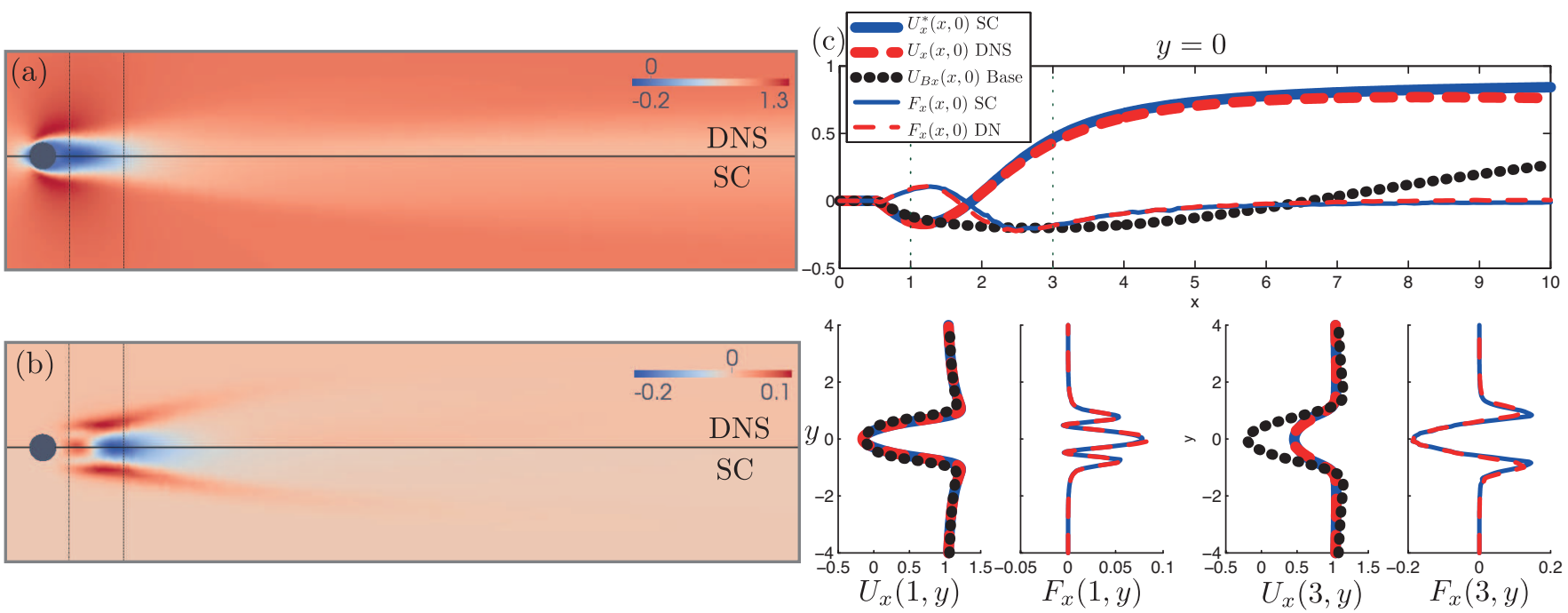

FIG. 2. (Color online) Comparison of the $x$-component of the mean flows (a) and the Reynolds stress divergence (b) for $R e=100$ computed from DNS (top half) and predicted from (5) (bottom half, SC). Plot (c) shows horizontal and vertical cuts for $\mathrm{y}=0$ (top), $\mathrm{x}=1$ (bottom left), and $\mathrm{x}=3$ (bottom right), for the $x$-component of the mean flows $\left(\boldsymbol{U}, \boldsymbol{U}^{*}\right)$, the base flow $\left(\boldsymbol{U}_{B}\right)$ and Reynolds stress divergence $\left(\boldsymbol{F}=\left\langle\left(\boldsymbol{u}^{\prime} \cdot \nabla\right) \boldsymbol{u}^{\prime}\right\rangle\right)$, as detailed in the legend.

amplitude $A$ is increased, the unstable mode turns the initial base flow into an increasingly modified mean flow through the divergence of the Reynolds stress in 4aal. In the process, the mean flow modifications simultaneously change the eigenmode structure and eigenvalue through the linearized perturbation equation (4b). One can expect that, from small to moderate values of the amplitude $A$, there exists a solution to the coupled perturbation-mean flow equations (4), meaning that the perturbation structure $\boldsymbol{u}_{1}$ is the one that forces the mean flow $\boldsymbol{U}$ by the Reynolds stress divergence in such a particular way that the mean flow generates the aforementioned perturbation structure $\boldsymbol{u}_{1}$. Associated to this solution there will be a certain growth rate $\sigma_{1}$ and frequency $\omega_{1}$, creating an implicit relation between the growth rate and amplitude $\sigma_{1}(A)$.

For a finite amplitude $A$, the software FreeFem ++ is used to discretize (4) and solve (4a) for a given $\boldsymbol{u}_{1}$, while SLEPC is used for the eigenvalue problem (4b) with a given $\boldsymbol{U}$. These two equations are then coupled through an iterative scheme until convergence is achieved. The eigenmode of an initial guess for the mean flow $\boldsymbol{U}_{g}$ (the base flow $\boldsymbol{U}_{B}$ or a solution of (4) for a smaller amplitude) is used for the Reynolds stress forcing in (4a), allowing to solve for a mean flow correction $\boldsymbol{U}_{c}$ which serves to generate a new guess $\boldsymbol{U}_{n g}=\gamma \boldsymbol{U}_{c}+(1-\gamma) \boldsymbol{U}_{g}$ where $0<\gamma<1$. The leading eigenmode of the new guess is then computed and the process is repeated until convergence is achieved. We have found that this procedure converges robustly provided the eigenmode is normalized by its associated Reynolds stress divergence, i.e. when $A_{f}^{2} \equiv A^{2}\left\|2 \Re\left(\left(\overline{\boldsymbol{u}}_{1} \cdot \nabla\right) \boldsymbol{u}_{1}\right)\right\|_{L^{2}} /\left\|\boldsymbol{u}_{1}\right\|_{L^{2}}^{2}$ is fixed. This is natural since variations of the amplitude $A_{f}$ directly con- trol the magnitude of the forcing term (RHS in 4a).

As shown in Fig. 1, the growth rate $\sigma_{1}$ resulting from these computations decreases as the amplitude of the Reynolds stress increases. At the same time, the structure of the Reynolds stress forcing computed from the most unstable eigenmode moves upstream with increasing amplitude $A_{f}$, (insets in Fig. 1) continuously modifying the mean flow and stabilizing it. The upstream migration of the Reynolds stress forcing follows the shortening of the recirculation region of the corresponding mean flow, indicated by the black line in each inset of Fig. 1, This length reduction of the recirculation region is totally in line with previous descriptions of the differences between the base and the mean flow [5, 11], and it has been proposed as a key feature for the instability saturation mechanism [18].

A clear physical picture emerges: the base flow $\boldsymbol{U}_{B}$ first develops its instability which grows and forces the underlying flow through the Reynolds stress divergence $2 A^{2} \Re\left(\left(\overline{\boldsymbol{u}}_{1} \cdot \nabla\right) \boldsymbol{u}_{1}\right)$, modifying it towards the mean flow. If one is to imagine that the evolution given by the NSE linearized around the model's mean flow (4b) could approximate the evolution of the vortex shedding around the exact mean flow, then one requires the leading eigenmode to oscillate in a purely sinusoidal way. This corresponds to the marginal stability criterion, consistent with the results of Barkley [5] and with the stabilizing effect of the growing perturbations on $\sigma_{1}(A)$ through the mean flow distortion (see Fig. 11). Therefore, we look for a particular amplitude $A^{*}$ for which there is a marginally stable mean flow $\boldsymbol{U}^{*}$ such that $\sigma_{1}^{*}\left(A^{*}\right)=0$. This amplitude $A^{*}$, which is not known a priori, will correspond to 
the saturation amplitude of the self-consistent model:

$$
\begin{aligned}
N\left(\boldsymbol{U}^{*}\right) & =-2 A^{* 2} \Re\left(\left(\overline{\boldsymbol{u}}_{1}^{*} \cdot \nabla\right) \boldsymbol{u}_{1}^{*}\right), \\
i \omega_{1}^{*} \hat{\boldsymbol{u}}_{1}^{*}+L_{\boldsymbol{U}^{*}}\left(\hat{\boldsymbol{u}}_{1}^{*}\right) & =0 .
\end{aligned}
$$

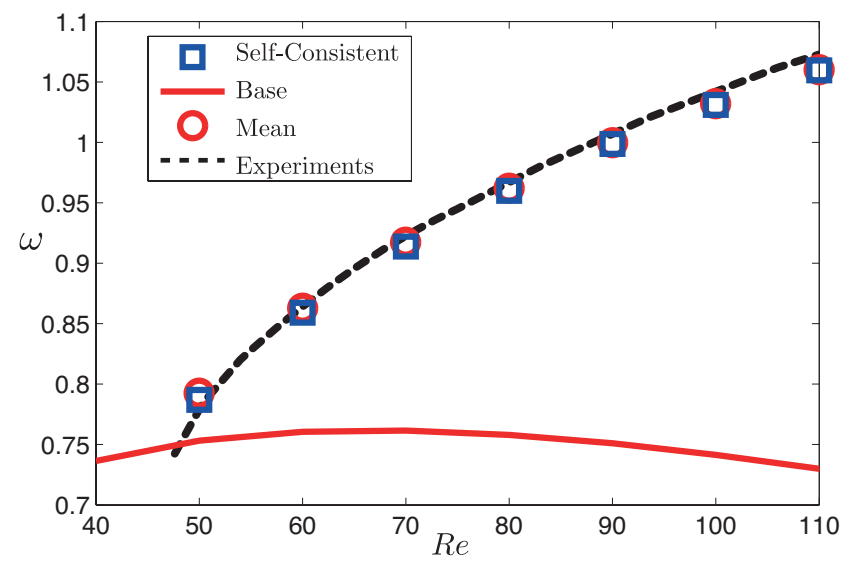

FIG. 3. (Color online) Comparison of the frequency predictions of the self-consistent model for different Reynolds numbers. Vortex shedding frequency from experiments by Williamson [12] (dashed black line), the present result (blue triangles), the most unstable mode of the base flow (solid red line) and the mean flow obtained from DNS (red squares).

Fig. 2(a) compares the converged mean flow $\boldsymbol{U}^{*}$ obtained by the present model (bottom half) with the mean flow of the nonlinear DNS (top half), showing that the approximation of the mean flow $x$-direction velocity is remarkable, with a length and minimum velocity of the recirculation region about $2 \%$ from the equivalent values of the full DNS. Moreover, the similarity of the Reynolds stress divergence of the model, calculated from the leading eigenmode $\boldsymbol{u}_{1}$, and that of the fully nonlinear timeaveraged DNS is striking (Fig. 20). A more quantitative comparison is given in Fig. 2(c). It should be highlighted that the full DNS Reynolds stress divergence is built by all the harmonics whereas in the self-consistent model it is constituted only by the leading eigenmode of the mean flow, which is approximatively neutrally stable. Both, the self-consistent and fully nonlinear Reynolds stress divergence present similar amplitude and spatial distribution concentrating the forcing close to the cylinder. The Reynolds stress forcing acts on their corresponding mean flows by pushing downstream the recirculation region of the base flow (Fig. 1) thus reducing its streamwise length, consistent with the recirculation length difference between the mean and the base flow [5, 11, 18].

Fig. 3 compares the frequency predicted by the present self-consistent saturation model with experimental and DNS data for different Reynolds number. Due to the ressemblance of the model and exact mean flows, it does not come as a surprise that the leading eigenfrequency of the present model falls onto the experimental data, as

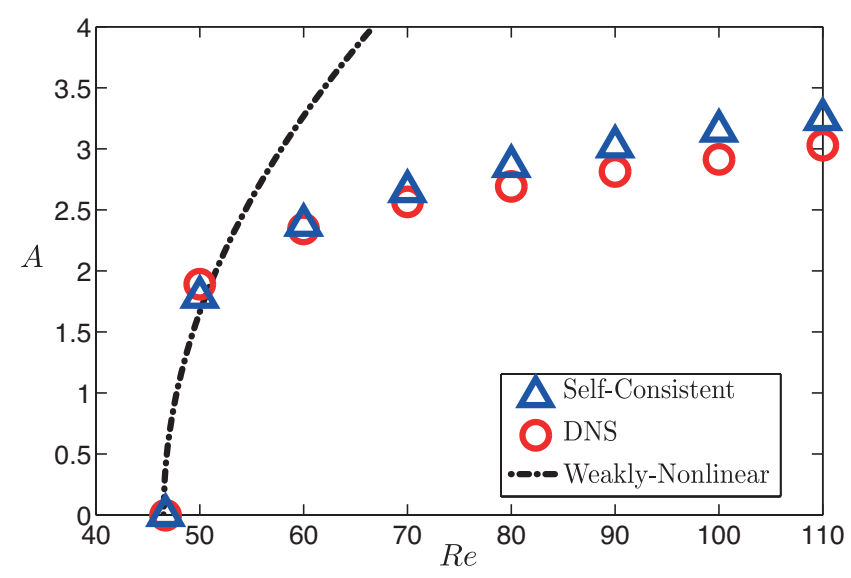

FIG. 4. (Color online) Comparison of the vortex shedding amplitude predictions of the self-consistent model for different Reynolds numbers. Saturation amplitude obtained from DNS (red circles), predicted by the self-consistent model (blue triangles) and as given according to weakly nonlinear expansion around threshold (dash-dotted line).

does the eigenfrequency of the linearly least stable mode of the real mean flow, as described by Barkley [5]. On the contrary, the unstable base flow considerably underestimates the experimental frequency.

The amplitude of the perturbation, defined as $A=$ $\left\|\boldsymbol{u}^{\prime}\right\|_{L^{2}}$, varying with the Reynolds number is compared in Fig. 团 for the DNS, self-consistent and the amplitude approximation of the weakly nonlinear theory. The weakly nonlinear theory is valid only close to the critical Reynolds number as it start to diverge from both the DNS and self-consistent results for $R e>50$. This is because the weakly nonlinear theory is based on a perturbative expansion around threshold [17], which is unsuitable to describe spatial variations of the oscillating mode [10] and yields an overestimated amplitude at saturation. In the self-consistent model this limitation is relaxed and the resulting amplitude follows the DNS results, indicating that the main nonlinear effects responsible for saturation are well captured in the coupling of the mean flow and perturbation equations in (5). As the Reynolds number increases, however, the number of iterations required for our direct method to converge to $\sigma_{1}^{*}=0$ becomes increasingly large. Similar issues for converging to steady solutions of the NSE when increasing Re have been reported in the literature, see 19 for instance.

The resolution of system (5) provides an excellent approximation of the mean flow velocity field and the perturbation's amplitude, frequency and spatial structure of the Reynolds stress forcing. Moreover, Eqs. (44) constitute a self-consistent model which formalizes and supports the idea of an instability saturation process wherein the perturbation, given by the most unstable eigenmode, 
grows around the mean flow and modifies it, saturating when the mean flow is marginally stable [5 -7], in a way reminiscent to Malkus [8] notions.

Note that some flows present positive growth rate when linear stability is computed around the mean flow 17], probably due to the neglected nonlinear terms in the perturbation equation (4) and the presence of higher harmonics. Nonetheless, the present model is expected to work for other laminar globally unstable flows dominated by a single harmonic and with a marginally stable mean flow. This includes flows reaching limit cycles above the bifurcation e.g. wakes, hot jets, mixing layers with counterflow, swirling jets, etc. but this excludes aperiodic, chaotic and turbulent flows. In addition, the model can be generalized to harmonic forcing response in stable cases. This can be done by applying a source term in the RHS of (5b) and replacing the unstable mode by the linear response to the forcing at a given frequency in (5a). This methodology can be used to include higher harmonics in the present case, adding linear equations for the higher harmonics as forced by the nonlinear interactions of the unstable mode.

The present quasi-linear self-consistent model is, for laminar flows, a deterministic counterpart of similar stochastic models recently developed to describe coherent structures in turbulent flows [20, 21]. It may open new possibilities as a model reduction for flow control [11], since the coupled mean flow-perturbation equations are solved as a closed system independent of time, allowing the calculation of a mean flow approximation a priori without requiring the full time evolution simulation for the a posteriori mean flow extraction.

[1] F. Charru, Hydrodynamic Instabilities (Cambridge University Press, 2011).

[2] G. S. Triantafyllou, M. S. Triantafyllou, and C. Chryssostomidis, J. Fluid Mech. 170, 461 (1986).

[3] D. A. Hammond and L. G. Redekopp, J. Fluid Mech. 331, 231 (1997)

[4] B. Pier, J. Fluid Mech. 458, 407 (2002).

[5] D. Barkley, Europhys. Lett. 75, 750 (2006).

[6] J. T. Stuart, J. Fluid Mech. 4, 1 (1958).

[7] A. Maurel, V. Pagneux, and J. E. Wesfreid, Europhys. Lett. 32, 217 (1995)

[8] W. V. R. Malkus, J. Fluid Mech. 1, 521 (1956).

[9] J. T. Stuart, J. Fluid Mech. 9, 353 (1960).

[10] J. Dusek, P. Le Gal, and P. Fraunié, J. Fluid Mech. 264, 59 (1994)

[11] B. R. Noack, K. Afanasiev, M. Morzynski, G. Tadmor, and F. Thiele, J. Fluid Mech. 497, 335 (2003).

[12] C. H. K. Williamson, Phys. Fluids 31, 2742 (1988)

[13] M. Provansal, C. Mathis, and L. Boyer, J. Fluid Mech. 182, 1 (1987)

[14] T. von Kármán, Göttingen Nachr. Math. Phys 12, 509 (1911)

[15] A. Zebib, J. Eng. Math. 21, 155 (1987).

[16] C. P. Jackson, J. Fluid Mech. 182, 23 (1987).

[17] D. Sipp and A. Lebedev, J. Fluid Mech. 593, 333 (2007)

[18] B. J. A. Zielinska, S. Goujon-Durand, J. Dusek, and J. E. Wesfreid, Phys. Rev. Lett. 79, 3893 (1997).

[19] B. Fornberg, J. Fluid Mech. 98, 819 (1980)

[20] B. F. Farrell and P. J. Ioannou, J. Fluid Mech. 708, 149 (2012)

[21] N. A. Bakas, and P. J. Ioannou, J. Fluid Mech. 740, 312 (2014) 